\title{
Noncontact Mapping-Guided Catheter Ablation of Atrial Fibrillation
}

\author{
Koichiro Kumagai, MD; Hideko Nakashima, MD
}

\begin{abstract}
Background An alternative approach to complete isolation of the posterior left atrium (LA), including all pulmonary veins (PVs), for treating atrial fibrillation (AF) is the Box isolation. However, it is sometimes difficult to confirm the conduction block within the linear lesion lines, so in the present study the efficacy of the Box isolation guided by noncontact mapping (NCM) was evaluated.

Methods and Results A total of 188 patients, 116 with paroxysmal, 48 with persistent and 24 with longstanding persistent AF, underwent the Box isolation using NCM system. The endpoint was defined as bi-directional conduction block in the posterior LA confirmed by an activation map using NCM during pacing inside and outside of the posterior LA. The induced atrial tachyarrhythmias and non-PV foci were also ablated using NCM. After $12 \pm 4$ months of follow-up, $91 \%$ of the patients with paroxysmal AF, $73 \%$ of those with persistent AF and $46 \%$ with longstanding persistent $\mathrm{AF}$ were arrhythmia-free without drugs.

Conclusions NCM is useful for defining complete Box isolation and detecting the conduction gaps, localizing non-PV foci, and analyzing the mechanism of atrial tachyarrhythmias. NCM-guided Box isolation is a feasible, safe and effective method of treating AF. (Circ J 2009; 73: 233-241)
\end{abstract}

Key Words: Ablation; Arrhythmia; Atrial fibrillation; Catheter ablation

$\mathbf{P}$ revious studies have demonstrated that the pulmonary veins (PVs) are a source of triggers that initiate and perpetuate atrial fibrillation (AF) 1,2 so radiofrequency catheter ablation that electrically isolates the PVs has been used to treat AF!-4 To improve the clinical outcome, additional linear ablation to modify the substrate of AF has been proposed ${ }^{5-7}$ and we recently described an alternative approach of complete isolation of the posterior left atrium (LA), including all the PVs, for treating AF, namely the Box isolation? However, this procedure is technically challenging because of variant atrial conductions, incomplete lesion lines or recovered conduction gaps being proarrhythmic.

\section{Editorial p 227}

Furthermore, during the procedure, non-PV foci can spontaneously occur, and atrial tachycardia or flutter can be induced after the Box lesion lines are completed. It is difficult to localize focal triggers and multiple conduction gaps, and analyze the mechanism of the atrial tachyarrhythmias with conventional point-to-point mapping. A noncontact mapping (NCM) system with a single-beat mapping capacity can facilitate the identification of the triggering sites of AF9-12 NCM allows fast recognition of the earliest depolarization sites, propagation patterns and conduction gaps, and also characterizes the relationship to the underlying anatomical structures. Therefore, we conducted this study to evaluate the feasibility and efficacy of the Box isolation using a NCM system.

(Received July 21, 2008; revised manuscript received August 20, 2008; accepted September 4, 2008; released online December 8, 2008) International University of Health and Welfare, Fukuoka, Japan

Mailing address: Koichiro Kumagai, MD, Department of Cardiology, Fukuoka Sanno Hospital, 3-6-40 Momochihama, Sawara-ku, Fukuoka 814-0001, Japan. E-mail: kumagai@kouhoukai.org

All rights are reserved to the Japanese Circulation Society. For permis-

sions, please e-mail: cj@j-circ.or.jp

\section{Methods}

\section{Patient Characteristics}

The study population consisted of 188 consecutive patients (166 men, 22 women; mean age $59 \pm 11$ years) with paroxysmal $(\mathrm{n}=116)$, persistent $(\mathrm{n}=48)$ and longstanding persistent $\mathrm{AF}(\mathrm{n}=24)$ who were referred for an electrophysiological study and catheter ablation. Persistent AF was defined as AF that was sustained beyond 7 days and requiring pharmacologic or electrical cardioversion. Longstanding persistent $\mathrm{AF}$ was defined as continuous $\mathrm{AF}$ of more than 1 year (mean, $91 \pm 69$ months, range 12-240 months). The mean LA diameter was $41 \pm 6 \mathrm{~mm}$ and the mean ejection fraction was $63 \pm 8 \%$ as determined by echocardiography. A mean of $3.0 \pm 1.0$ antiarrhythmic drugs had been administered, but failed to maintain normal sinus rhythm. None of the patients had been treated with amiodarone within the 6 months preceding the procedure. No structural heart disease was present in 65 patients. Valvular disease was documented in 22 patients, cardiomyopathy in 8, coronary artery disease in 8 , and sick sinus syndrome in 2 . Hypertension was present in 83 patients. Written informed consent was given by all patients.

\section{Electrophysiological Study}

All patients received oral anticoagulants for at least 1 month before the ablation. Transesophageal echocardiography was performed to exclude any LA thrombi and antiarrhythmic drugs were discontinued for 5 half-lives before the procedure. Three 5-Fr quadripolar electrode catheters (St Jude Medical (SJM), St Paul, MN, USA) were placed in the high right atrium, His bundle area, and coronary sinus (CS). Triple transseptal punctures were performed, and two 8-Fr SL0 sheaths (SJM) and a 10-Fr SL0 sheath (SJM) were advanced into the LA. After the transseptal puncture, $100 \mathrm{IU}$ heparin/kg was given intravenously and heparinization was 
continued during the procedure to maintain an activated clotting time $>300 \mathrm{~s}$. Angiography was performed from a sheath to determine the size and shape of the PVs. Patients were asked to swallow $5 \mathrm{ml}$ of barium sulfate and the location of the esophagus was noted by fluoroscopy. The surface electrocardiogram (ECG) and bipolar endocardial electrogram were continuously monitored and stored on a computer-based digital amplifier/recorder system for offline analysis (LabSystem ${ }^{\mathrm{TM}}$ PRO, Bard Electrophysiology, Lowell, MA, USA). The intracardiac electrograms were filtered from 30 to $500 \mathrm{~Hz}$ and measured at a sweep speed of $200 \mathrm{~mm} / \mathrm{s}$. Atrial pacing was performed using a programmed stimulator (SEC-3102, Nihon Kohden, Tokyo, Japan).

\section{$N C M$}

A multielectrode array catheter (EnSite 3000; SJM) was inserted into the LA via the 10-Fr SLO sheath and the tip of the array was positioned in the LA appendage. A decapolar ring catheter (Lasso, Biosense-Webster, Diamond Bar, CA, USA) was inserted into the LA and used for mapping of the PVs. A deflectable, quadripolar 7-Fr ablation catheter (Ablaze Fantasista, Japan lifeline Co, Ltd, Tokyo, Japan) was also inserted into the LA for mapping and ablation. The NCM technique has been described elsewhere! ${ }^{1-15}$ The 3-dimensional geometry was created by maneuvering the Lasso and ablation catheters in the LA and PVs under fluoroscopic and angiographic guidance.

In patients who had been in sinus rhythm at the beginning of the study, derived isopotential and isochronal activation maps were analyzed during sinus rhythm, pacing from the proximal CS, and pacing from 5 sites (left superior, left inferior, center, right superior, and right inferior) within the posterior LA. Burst pacing was then performed in an attempt to induce sustained AF prior to proceeding with ablation.

The conduction block was defined as no propagation of wavefront across the line, and virtual unipolar electrograms along the line showed 2 discrete deflections in reversed polarity and separated by an isoelectric baseline.

\section{Catheter Ablation}

Ablation was performed with a temperature-controlled, dumbbell-shaped 8-mm-tip ablation catheter (Ablaze Fantasista, Japan lifeline). In 153 patients (81\%) with spontaneous or induced sustained $\mathrm{AF}$ and persistent $\mathrm{AF}$, ablation was performed in either induced sustained or persistent AF; in the other 35 patients, ablation was performed during sinus rhythm.

Radiofrequency energy was delivered with a power of $30-40 \mathrm{~W}$. The temperature was limited to $50^{\circ} \mathrm{C}$. When radiofrequency energy was delivered at the anterior aspect of the left PVs and the floor line near the esophagus, the power was reduced to $30 \mathrm{~W}$. Radiofrequency energy was delivered for $30 \mathrm{~s}$ at each site, and that application was prolonged for 60 s when a change in the morphology or sequence of the PV potentials occurred as determined by circumferential mapping. When the floor line crossed close to the esophagus, the ablation was performed at a maximum power of $30 \mathrm{~W}$ and a temperature of $45^{\circ} \mathrm{C}$ for $15 \mathrm{~s}$.

Continuous lesions in the anterior portion of the ipsilateral superior and inferior PVs were initially created under guidance of the Lasso catheter and NCM system. Ablation was started at the superior wall and continued around the anterior and inferior venous perimeter. When ablation was required in the anterior portion of the left PVs, the energy was delivered within the most proximal millimeters of the vein ostium (rather than the posterior wall of the appendage) to achieve effective electrical disconnection. There was no vertical lesion line created in the posterior portion of the PVs along the esophageal aspect of the posterior LA.

Ablation of the LA roof was then performed by creating a contiguous line of ablation lesions joining the superior PVs. Commencing at the lesion at the left superior PV, the sheath and catheter were rotated clockwise posteriorly and dragged toward the right superior PV. Local potential elimination or the formation of double potentials signified the local effectiveness of the ablation. The stability of the catheter was monitored during the radiofrequency applications by the proximal electrograms, real-time monitoring by NCM and intermittent fluoroscopy to recognize inadvertent displacement of the catheter. The radiofrequency applications were tagged on the NCM system.

Finally, ablation of the LA floor was performed by creating a contiguous line of ablation lesions joining the inferior PVs in order to isolate the posterior LA? Commencing from the lesion at the left inferior PV, the sheath and catheter were rotated clockwise posteriorly and dragged toward the right inferior PV. If AF did not terminate after the Box isolation, sinus rhythm was restored by transthoracic cardioversion. Box isolation was evaluated with NCM after the restoration of sinus rhythm to allow pacing from the CS, posterior LA and all PVs. Once residual conduction gaps were identified, additional radiofrequency applications were applied till all the gaps were eliminated.

The endpoint of the Box isolation was defined as all PV isolations indicated by circumferential PV mapping and bidirectional conduction block in the posterior LA confirmed by activation maps using NCM during pacing at output of $5 \mathrm{~V}$ both inside (the 5 sites) and outside (proximal CS and sinus rhythm propagation) of the posterior LA.

After the Box isolation, the inducibility of AF was evaluated by rapid atrial pacing (output, $5 \mathrm{~V}$ ) performed ( 20 bursts) in decrements from a cycle length of $250 \mathrm{~ms}$ down to refractoriness from the high right atrium and CS after the infusion of isoproterenol $(10-20 \mu \mathrm{g} / \mathrm{min})$. Inducible AF was defined as sustained $\mathrm{AF} \geq 5 \mathrm{~min}$. Sinus rhythm was restored by cardioversion in those patients with sustained AF. If atrial tachycardia/flutter was induced, activation mapping with NCM and pacing entrainment were performed to characterize the arrhythmias and facilitate their ablation followed by terminating them. If the earliest activation site in the LA revealed a positive $\mathrm{R}$ wave on the noncontact unipolar electrogram, it suggested RA origin and the multielectrode array was moved to the RA.

\section{Post-Ablation Care and Follow-up}

Intravenous heparin was administered to all patients for $24 \mathrm{~h}$ after the procedure, followed by warfarin for 3 months. After the ablation, all antiarrhythmic drugs were discontinued. On the day following the procedure, surface ECG, transthoracic echocardiography, and 24-h Holter recording were performed and repeated at 1, 3, 6, and 12 months. All patients were provided with a telemetry ECG recorder (Omron HCG-801, Kyoto, Japan) for 1 month at 6 months after ablation to document symptomatic episodes or to record an ECG once per week if asymptomatic. Any episode of symptomatic or asymptomatic atrial tachyarrhythmia, which was identified on the surface ECG, Holter monitoring or telemetry ECG recorder and lasted for more than $30 \mathrm{~s}$, was considered a recurrence. The follow-up was immediately started after the procedure without any blanking or break- 
A

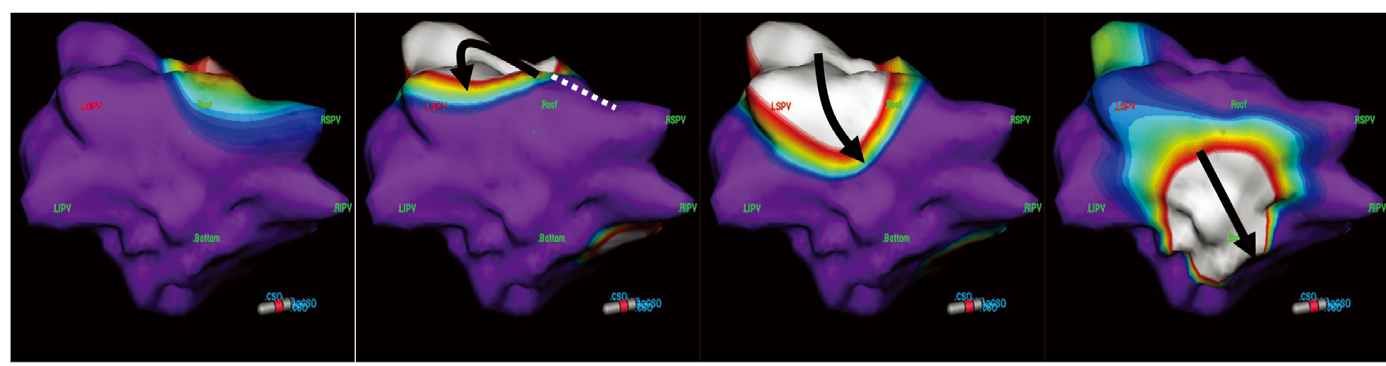

B

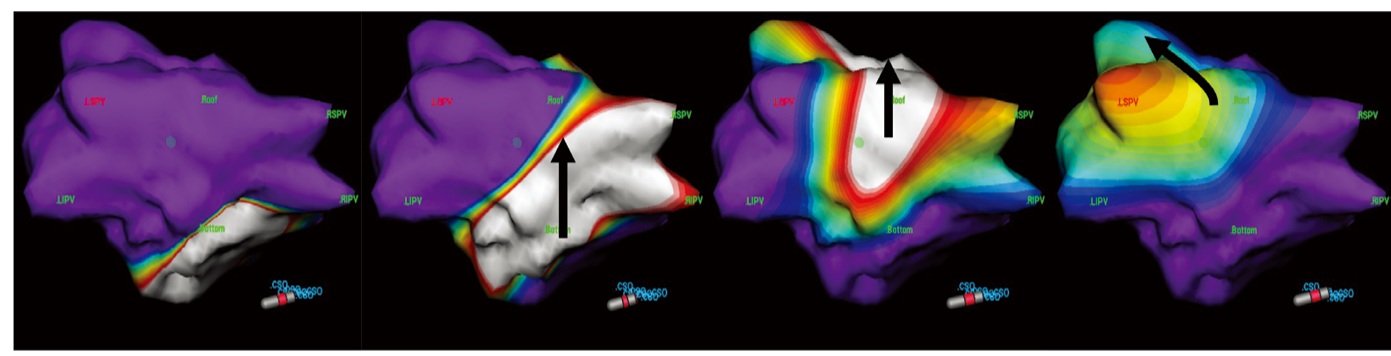

C

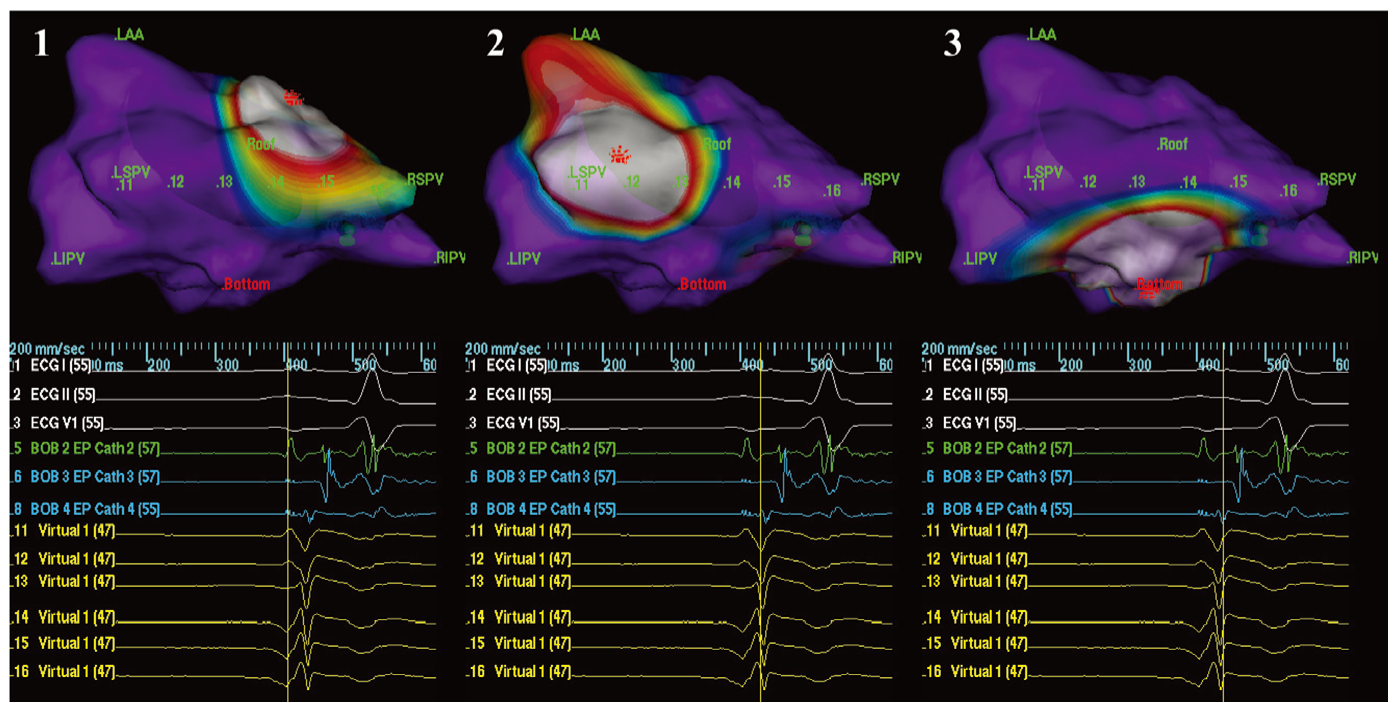

Fig 1. (A) Posterior view of an activation map using noncontact mapping (NCM) during sinus rhythm. White represents the peak negative voltage of the local virtual unipolar potentials. The voltage color range (red, yellow, green, or blue) is set according to the voltage amplitude. A wavefront coming from the anterior septum propagated to the posterior left atrium (LA) through the left side of the LA roof. The white dot line at the right side of the LA roof indicates a functional block. (B) Activation map using NCM during coronary sinus (CS) proximal pacing. The pacing impulse propagated from the LA floor to the roof. (C) NCM and virtual unipolar electrograms of the LA roof. Virtual 11-16 match the markings of 11-16 on the map. In contrast to the RS pattern observed in the unipolar electrograms at 11 and 12, 13-16 reveal the QSRS pattern with polarity reversal. Thus, a functional block line is thought to be present at 13-16. LAA, left atrial appendage; LSPV, left superior pulmonary vein; RSPV, right superior pulmonary vein; LIPV, left inferior pulmonary vein; RIPV, right inferior pulmonary vein.

ing period in the present study. In the event of recurrent symptomatic or asymptomatic arrhythmias, the patients were offered an additional ablation after a trial of drug therapy. During the repeated procedures, the prior ablation lesions were evaluated and re-established as required. Cessation of anticoagulant therapy was considered after 3 months if the patient remained in sinus rhythm.

\section{Statistical Analysis}

All values are expressed as the mean \pm SD. The compari- sons of echocardiographic parameters and B-type natriuretic peptide (BNP) level between that before the ablation and 3 months after were calculated using a paired Student's t-test. Statistical significance was set at $\mathrm{P}<0.05$.

\section{Results}

Activation Patterns of the Posterior LA Prior Ablation

The LA activation map from the NCM during sinus rhythm was analyzed in 57 patients. In sinus rhythm, LA 
A

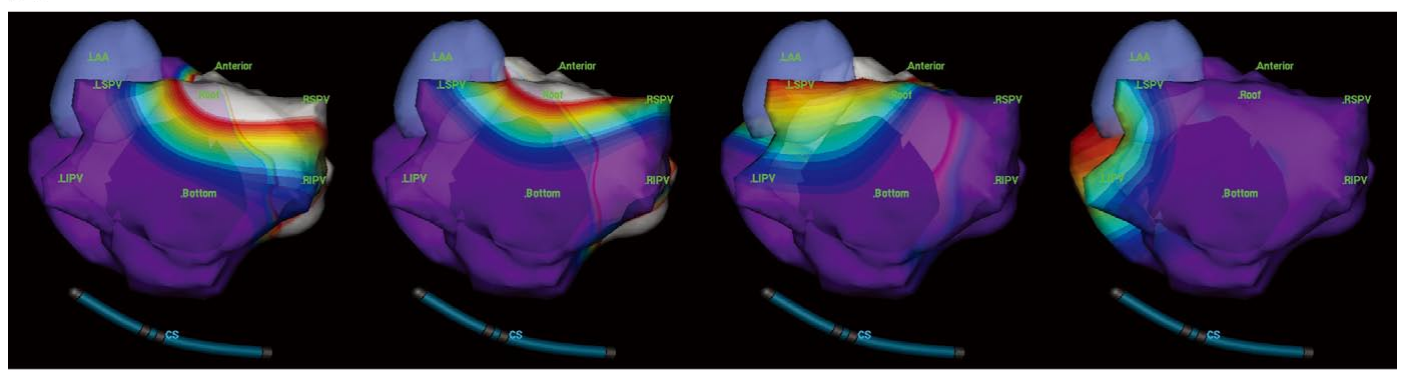

B

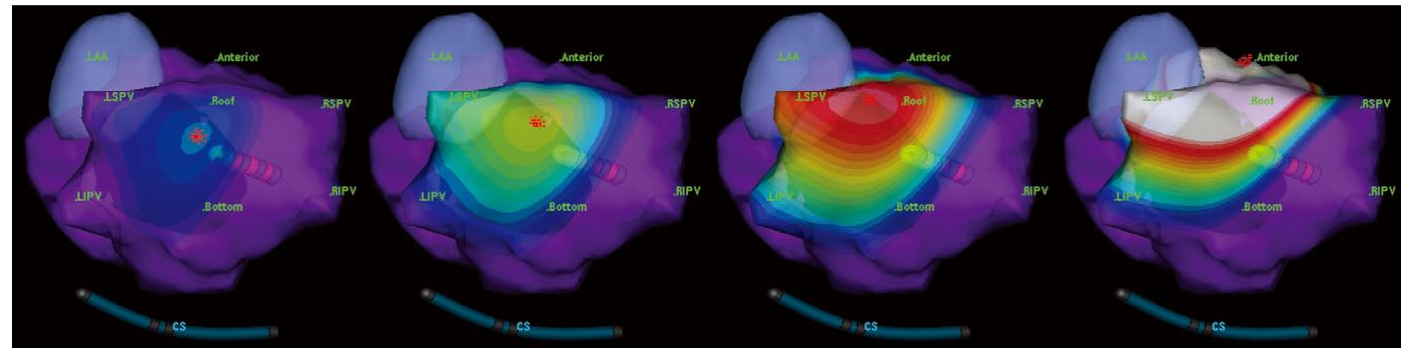

Fig 2. Unidirectional conduction block of the posterior LA. (A) The posterior view of an activation map using NCM during sinus rhythm. A wavefront coming from the anterior septum did not propagate to the posterior LA along the LA roof. (B) Activation map using NCM during pacing from the posterior LA. The pacing impulse propagated to the LA roof. Abbreviations see in Fig 1.

A

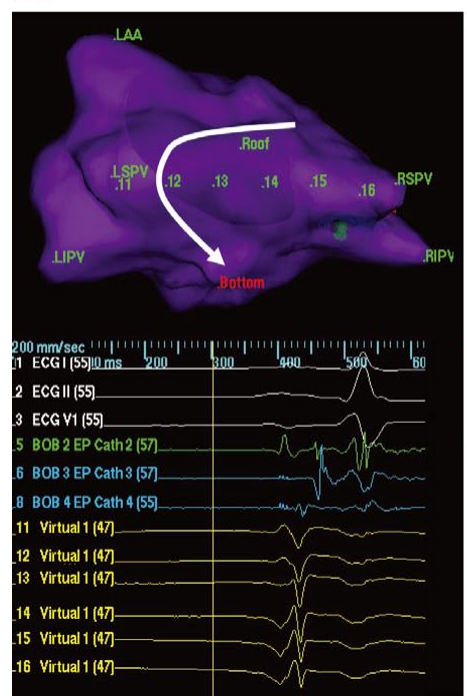

B

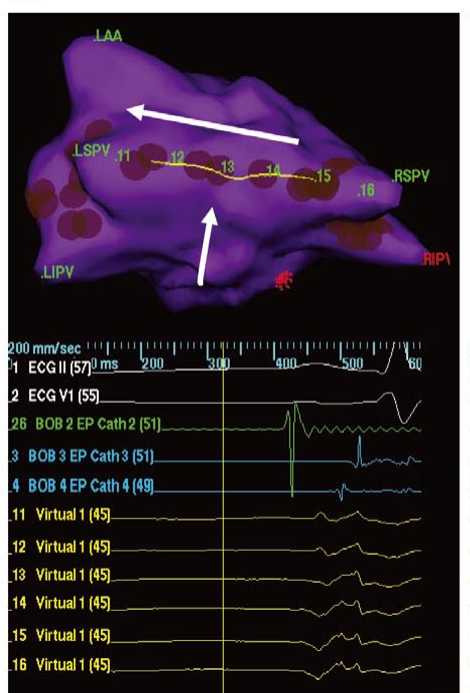

C

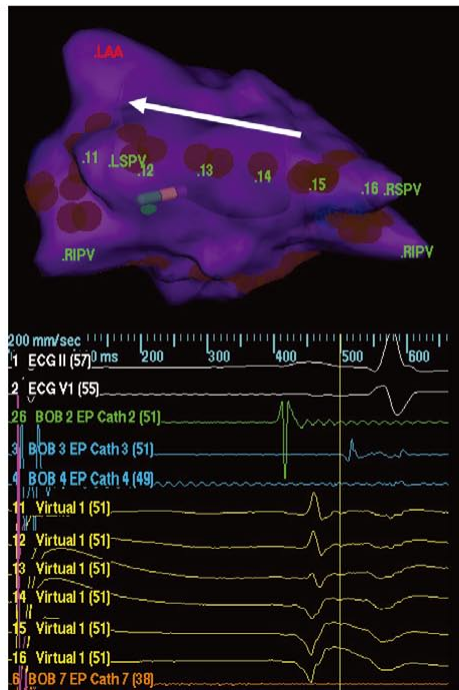

Fig 3. Evidence of entrance block in the posterior LA. (A) NCM and virtual unipolar electrograms of the LA roof before ablation (panel A is the same as Fig 1C). (B) After roof ablation, the second component of the double potentials was positive, indicating a wavefront from the LA floor to the roof. (C) After floor ablation, the second component disappeared because there was not a wavefront coming from the LA floor. Abbreviations see in Fig 1.

activation was characterized by the presence of a consistent, but varying, functional line of conduction block that extended from the LA roof, descended the posterior LA wall between the ostia of the superior PVs and then between the ostia of the inferior PVs, and passed below the ostium of the RIPV (Figs 1A,C). A functional line of conduction block of the LA roof was observed in 34 (60\%) of the 57 patients.

During proximal CS pacing, LA activation initiated near the mitral annulus and propagated upward at the posterior LA, in a reverse sequence to that in sinus rhythm, and the latest activation was at the roof to the left superior PV ostium (Fig 1B). A functional line of conduction block of LA floor was observed in $39(68 \%)$ of the 57 patients.

During pacing inside the pre-designed Box lines at the posterior LA, conduction from the inside to the outside of the posterior LA through the LA roof was observed in 55 $(96 \%)$ of the 57 patients, and through the LA floor was seen in $52(91 \%)$ of the 57 patients. In $35(90 \%)$ of the 39 patients who had a conduction block at the LA roof or floor in sinus rhythm or with proximal CS pacing, the conduction 
A

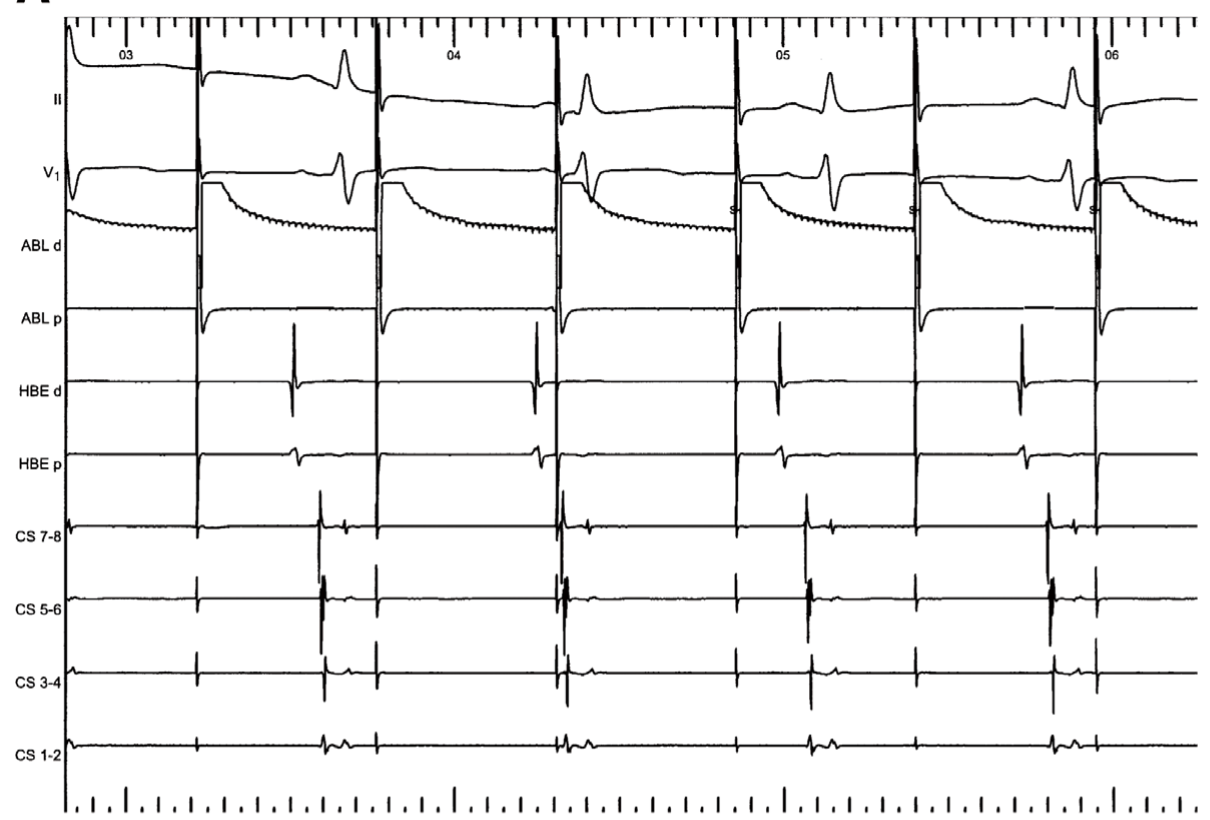

B

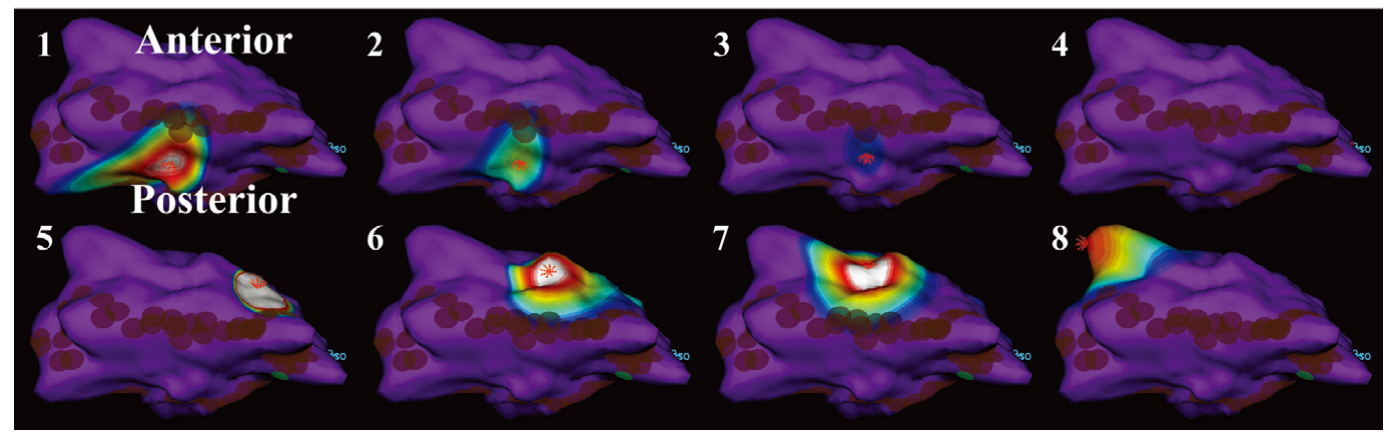

Fig 4. Evidence of a bi-directional conduction block of the posterior LA. (A) Electrocardiogram (ECG) and intracardiac electrograms during pacing from the posterior LA. The pacing from inside the box could not capture outside the box. (B) Activation map using NCM during pacing from the posterior LA after the Box isolation. An impulse from pacing inside the box did not propagate to the outside of the box (Panels 3-4), and the propagation wavefront from sinus rhythm did not propagate to the inside the box (Panels 5-8). The brown dots represent the radiofrequency ablation markers. ABL, ablation catheter; HBE, His bundle electrogram; p, proximal; d, distal. Other abbreviations see in Fig 1.

blocks were reversed during posterior LA pacing (Fig 2), which indicated that the majority of conduction blocks observed in both sinus rhythm and CS pacing were functional blocks.

\section{Box Isolation}

Entrance block of the posterior LA during sinus rhythm and proximal CS pacing was achieved in all patients (Fig 3), whereas the exit block of the posterior LA during pacing from inside the box area was achieved in 173 patients $(92 \%)$. Therefore, bi-directional conduction block of the posterior LA was completed in 92\% of the patients (Fig 4). In the remaining patients, complete Box isolation could not be achieved because of incomplete block of the roof or floor line, but all PVs were isolated.

The mean duration of the radiofrequency energy applications was $50 \pm 16 \mathrm{~min}$, and the mean procedure and fluoroscopy times were $152 \pm 23$ and $31 \pm 18 \mathrm{~min}$, respectively.
Termination of AF During Radiofrequency Applications

Of 83 patients with paroxysmal AF in whom ablation was performed during $\mathrm{AF}$, termination of the $\mathrm{AF}$ by ablation was achieved in 51 patients $(61 \%)$. In the remaining patients, AF was converted to sinus rhythm by transthoracic cardioversion.

Among 48 patients with persistent $\mathrm{AF}$, the $\mathrm{AF}$ was terminated by ablation in 7 patients (15\%), and sinus rhythm was restored by transthoracic cardioversion in the remaining patients.

In patients with longstanding persistent $\mathrm{AF}$, none had the $\mathrm{AF}$ terminated by radiofrequency ablation.

\section{Atrial Tachyarrhythmias Induced After Box Isolation}

After the Box isolation, AF was no longer inducible in $74 \%$ of the patients with paroxysmal AF, in $66 \%$ of those with persistent $\mathrm{AF}$ and in $43 \%$ with longstanding persistent AF. With rapid atrial pacing, atrial tachyarrhythmias were induced in 51 patients (27\%). Activation using NCM combined with entrainment mapping in those patients demon- 
A

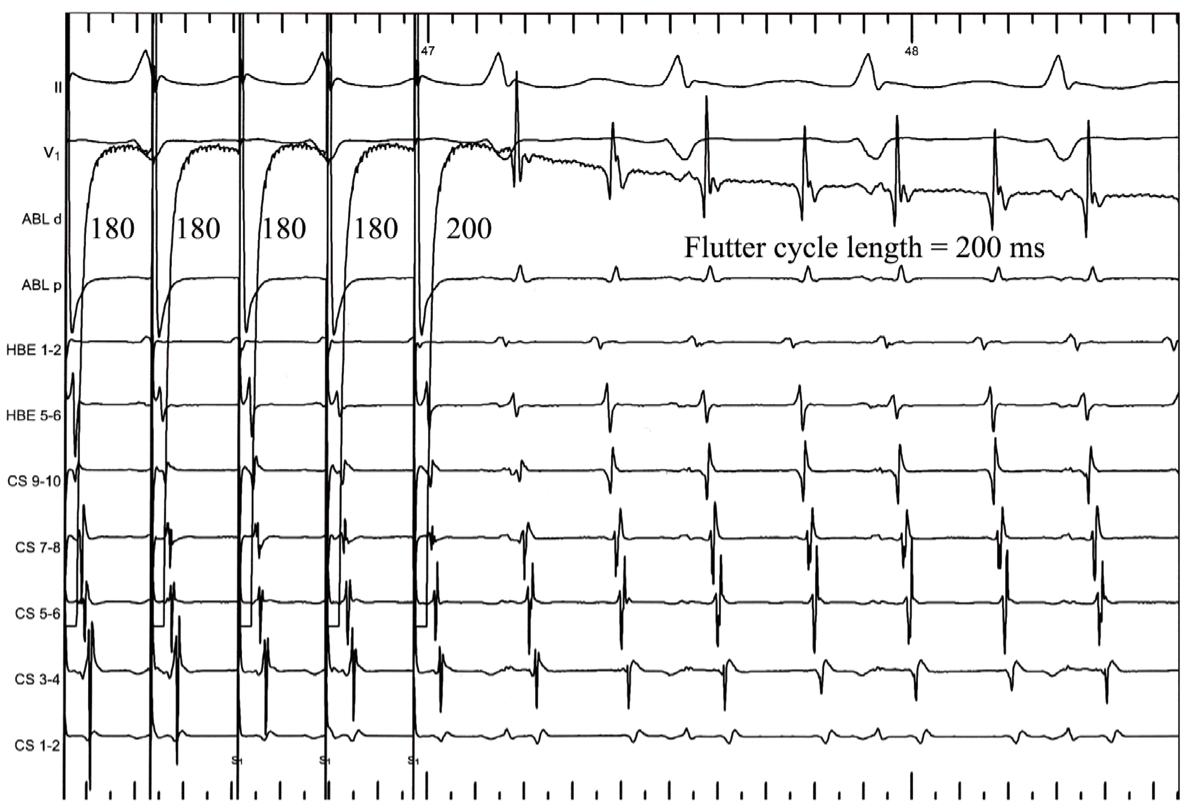

B

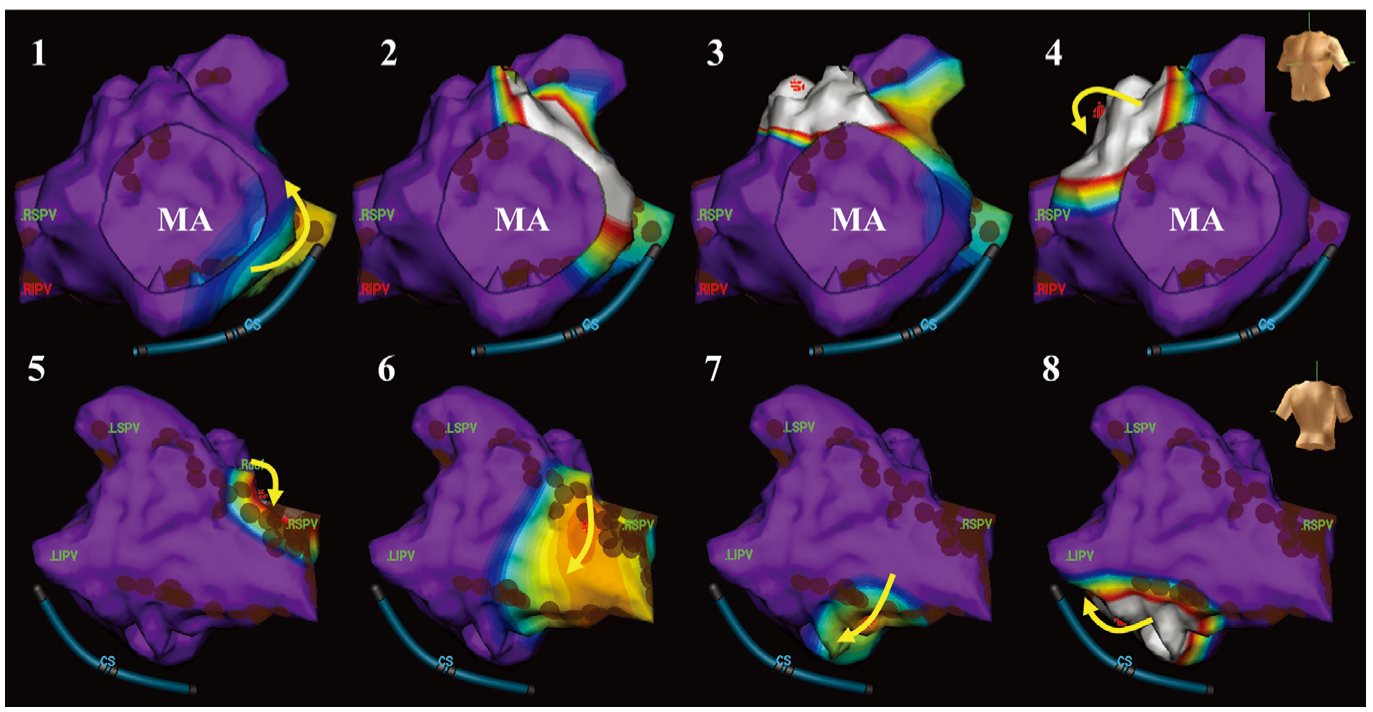

Fig 5. Macroreentrant LA flutter. (A) ECG and intracardiac electrograms during atrial flutter induced after the Box isolation. Entrainment was confirmed by pacing from the inferior LA near the gap in the floor line. Post-pacing interval was the same as the flutter cycle length. (B) Anterior (Panels 1-4) and posterior views (Panels 5-8) of an activation map using NCM during atrial flutter. The map reveals macroreentry that utilized the conduction gaps in the roof of the RSPV and the mid floor line. A wavefront went into the box from the roof of the RSPV and went out of the box from the mid floor line. MA, mitral annulus. Other abbreviations see in Figs 1,4.

strated macroreentrant LA flutter that utilized the conduction gaps in the linear lesions (Fig 5) or mitral annulus in 44 patients, and focal atrial tachycardia originating from the anterior LA wall in 5 and the RA in 2. Those atrial tachyarrhythmias were successfully ablated by additional ablation applications targeted to the gaps, mitral isthmus or foci. The cavotricuspid isthmus was also successfully ablated during the procedure in 25 patients who had typical atrial flutter documented prior to the procedure or induced by pacing after the Box isolation. The superior vena cava was also isolated in 12 patients who had focal AF spontaneously occurring from that site after the Box isolation.

\section{Outcome After Initial Ablation Procedure}

During a mean follow-up of $9 \pm 4$ months after a single ablation procedure, $101(87 \%)$ of the 116 patients with paroxysmal AF, $33(69 \%)$ of the 48 patients with persistent $\mathrm{AF}$ and $10(42 \%)$ of the 24 patients with longstanding persistent $\mathrm{AF}$ were arrhythmia-free without any antiarrhythmic drugs. The remaining patients who had recurrent $\mathrm{AF}$ were receiving medical treatment.

Repeat Ablation A second ablation procedure was performed at a mean of $7 \pm 4$ months after the first procedure for those patients with symptomatic, drug refractory recurrent AF: 4 (3\%) patients with paroxysmal AF, 2 (4\%) with persistent $\mathrm{AF}$ and $2(8 \%)$ with longstanding persistent $\mathrm{AF}$ who 
A

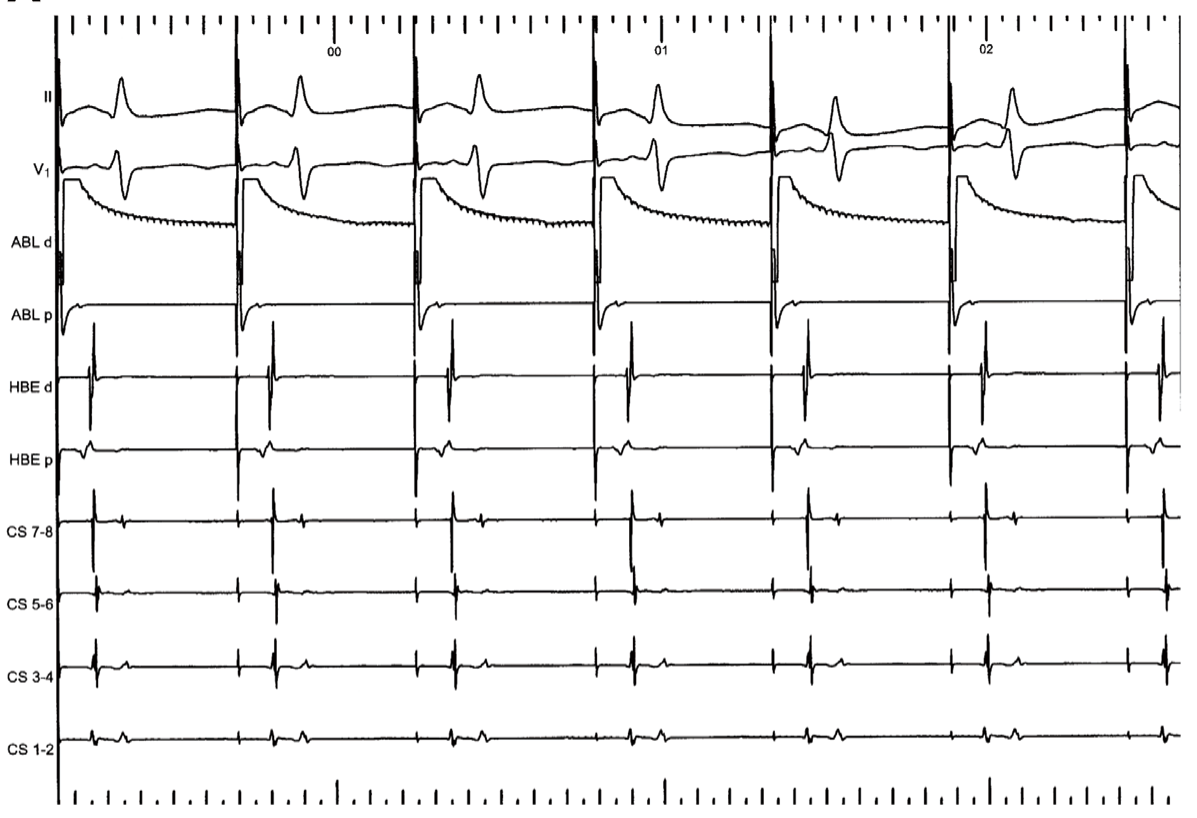

B

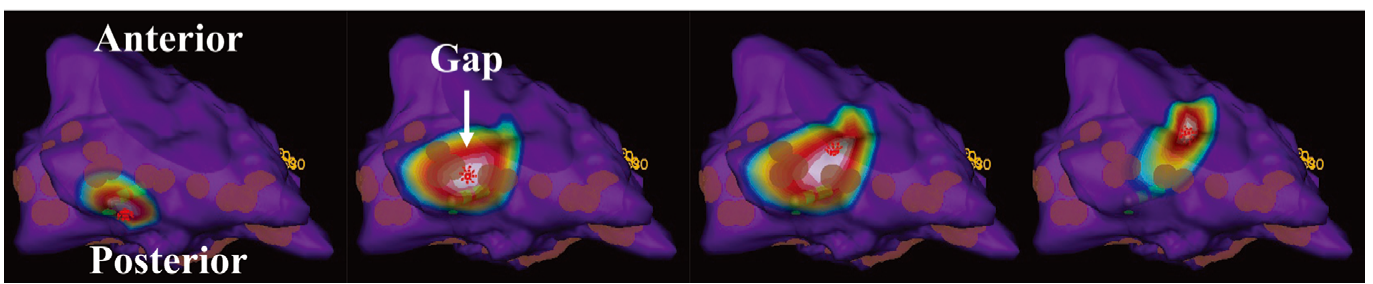

Fig 6. Identification of a conduction gap. (A) ECG and intracardiac electrograms show that the pacing inside the box captures outside the box. (B) Activation map using NCM during pacing from the posterior LA. The pacing impulse propagated to the LA roof through a gap in the mid roof. Abbreviations see in Figs 1,4.

had AF that did not respond to antiarrhythmic drugs. During the procedure, recovered conduction gaps were found in the left-sided PV line in 5, in the right-sided PV line in 4, in the roof line in 4 (Fig 6), and in the floor line in 4 . In 1 patient, $\mathrm{AF}$ recurred because of a focal trigger from the superior vena cava. All conduction gaps were successfully closed by segmental radiofrequency applications. For the patient with focal AF originating from the superior vena cava, an isolation procedure was performed.

In the remaining patients with recurrent $\mathrm{AF}, 28$ were $\mathrm{AF}$ free with antiarrhymic drug therapy, and 8 were asymptomatic AF with drugs and did not undergo a second procedure.

\section{Overall Outcome of NCM-Guided Box Isolation}

During a mean follow-up of $12 \pm 4$ months (range 6-18 months) after the final ablation procedure, $105(91 \%)$ of the 116 patients with paroxysmal AF, $35(73 \%)$ of the 48 with persistent $\mathrm{AF}$ and $11(46 \%)$ of the 24 patients with longstanding persistent AF were arrhythmia-free without antiarrhythmic drugs, including the 8 patients who underwent a second procedure. In addition, 8 patients with paroxysmal $\mathrm{AF}, 10$ with persistent $\mathrm{AF}$ and 10 with longstanding persistent AF were arrhythmia-free with antiarrhythmic drugs.

Ablation significantly reduced the mean LA diameter ( $41 \pm 6 \mathrm{~mm}$ vs $39 \pm 6 \mathrm{~mm}, \mathrm{P}<0.01$ ), increased the mean ejection fraction $(63 \pm 8 \%$ vs $68 \pm 7 \%, \mathrm{P}<0.05)$ and decreased the BNP level $(72 \pm 103 \mathrm{pg} / \mathrm{ml}$ vs $26 \pm 28 \mathrm{pg} / \mathrm{ml}, \mathrm{P}<0.01)$

\section{Complications}

Pericardial effusion occurred after the first procedure in 1 patient and was managed by percutaneous drainage. One patient developed a groin hematoma during the initial procedure. No esophageal injuries or significant PV stenosis or stroke occurred.

\section{Discussion}

\section{Main Findings}

In the present study, NCM was safely and effectively used to guide and confirm a bi-directional conduction block of the posterior LA in a complete Box isolation procedure for treating AF. After the ablation, atrial tachyarrhythmias were induced in $27 \%$ of the patients. NCM combined with pacing maneuvers can identify the mechanism of atrial tachyarrhythmias and conduction gaps. A successful second ablation procedure guided by NCM was performed in those patients with drug-resistant, symptomatic recurrent $\mathrm{AF}$. With the complete Box isolation, $91 \%$ of the patients with paroxysmal AF, $73 \%$ of those with persistent AF and $46 \%$ with longstanding persistent AF were arrhythmia-free without antiarrhythmic drugs. 


\section{Characteristics of the LA Activation}

Markides et al analyzed the activation maps with a NCM system during initiation of $\mathrm{AF}$ and observed the interaction between wavefronts entering the LA from the PVs and a functional line of conduction block that resulted in LA macroreentry or formation of daughter wavefronts 16 Weber et al also investigated the onset of episodes of paroxysmal AF in the LA using NCM, and identified that rapid break up and disorganization of wavefronts, particularly on the posterior wall, could maintain AF without the need for a repetitive firing focus $!^{17}$ In the present study, a functional line of conduction block of the LA roof was observed in $60 \%$ of the patients during sinus rhythm, in the LA floor in $68 \%$ during CS pacing, and 63\% of such conduction blocks of the LA roof or floor could be reversed by posterior LA pacing. Thus, the conduction properties of the posterior LA are changed by the direction of the wavefronts, and that may have played a role in the initiation and perpetuation of the AF.

\section{Usefulness of NCM}

During catheter ablation of AF, it is sometimes difficult to identify focal triggers, confirm the conduction block of the linear lesions, and analyze the mechanism of atrial flutter associated with the procedure. The NCM system is a useful tool for mapping and guiding the ablations at trigger sites for AF initiation with its single-beat mapping capacity! ${ }^{12-12,18}$ In the present study, NCM effectively detected the conduction gaps in the linear lesions, localized non-PV foci, and analyzed the mechanism of the flutter. Therefore, complete isolation of the posterior LA was achieved in most of the AF patients, and may have resulted in the overall higher success rate. Moreover, the NCM system can provide real-time catheter navigation during the ablation and decrease the use of fluoroscopy. No complications, such as a thrombus formation, occurred in the present study, so NCM can be safely used in the LA.

\section{Efficacy of the Box Isolation}

We have previously reported the feasibility and efficacy of the Box isolation for treating paroxysmal $\mathrm{AF}^{8} \mathrm{In}$ the present study, in addition to the patients with paroxysmal AF, we performed the NCM-guided Box isolation in patients with persistent or longstanding persistent $\mathrm{AF}$, and the success rate of maintaining normal sinus rhythm without any antiarrhythmic drugs was $91 \%, 73 \%$ and $46 \%$, respectively. Monsour et al performed a Fourier analysis of the bi-atrial fibrillatory signals in a sheep model of AF and found that the fastest frequencies recorded during $\mathrm{AF}$ occurred in the posterior LA, and that a left-to-right atrial frequency gradient existed during $A F{ }^{19}$ This important finding suggests that the posterior LA and $\mathrm{PV}$ region play a role in maintaining $\mathrm{AF}$. Lazor et al later confirmed that this left-to-right atrial dominant frequency gradient exists during human paroxysmal $\mathrm{AF}$, but was attenuated during longer-lasting persistent $\mathrm{AF}^{20}$ which suggests that, although the posterior LA and $\mathrm{PV}$ regions are important for maintaining paroxysmal $\mathrm{AF}$, other regions outside the posterior LA may play a role in maintaining persistent AF. Therefore, additional ablation at sites with complex fractionated atrial electrograms outside the posterior LA area may improve the clinical outcome ${ }^{21}$

Another interesting finding from this study is that Box isolation shows an improved outcome for patients with recurrent $\mathrm{AF}$ and previous failure of antiarrhythmic drug treatments. A repeat ablation procedure was needed and per- formed in only $8 \%$ of that group of patients, so we believe that a hybrid therapy of the Box isolation and antiarrhythmic drugs is feasible and effective for longstanding persistent $\mathrm{AF}$.

\section{Study Limitations}

Previous studies have validated the accuracy of using NCM to map areas of interest during sinus rhythm and AF in humans, and it is well known that its accuracy is limited by the distance to the array center ${ }^{2-24}$ The accuracy of reconstructed electrograms is decreased at a distance $>40 \mathrm{~mm}$ from the center of the array, particularly during $\mathrm{AF}^{22}$ In the present study group of AF patients, the average parasternal long-axis LA diameter was only $41 \pm 6 \mathrm{~mm}$ on transthoracic echocardiography. However, because of the eccentric geometry of the LA, the septum-to-lateral wall diameter was much greater than the measurement and therefore the PVs may be $>40 \mathrm{~mm}$ from the center of the array. To minimize this distance factor, we used a Lasso catheter to map the PVs and confirm the elimination of PV potentials during sinus rhythm. Another limitation is that our observation of functional blocks at the posterior LA was based on the group of $\mathrm{AF}$ patients. A future comparative study in non-AF patients may further benefit its definition.

\section{Conclusions}

There were functional lines of conduction block at the posterior LA in most of this group of AF patients. NCM was useful for guiding a complete Box isolation and detecting the conduction gaps, localizing non-PV foci, and analyzing the mechanism of flutter. The success rate of Box isolation in a single procedure is high, and the need for repeat procedures is low. Thus, NCM-guided Box isolation is a safe, feasible and effective method of treating AF.

\section{Disclosures}

All authors report no disclosures or conflicts.

\section{References}

1. Haissaguerre M, Jais P, Shah DC, Takahashi A, Hocini M, Quiniou $\mathrm{G}$, et al. Spontaneous initiation of atrial fibrillation by ectopic beats originating in the pulmonary veins. N Engl J Med 1998; 339: 659666.

2. Chen SA, Hsieh MH, Tai CT, Tsai CF, Prakash VS, Yu WC, et al. Initiation of atrial fibrillation by ectopic beats originating from the pulmonary veins: Electrophysiological characteristics, pharmacological responses, and effects of radiofrequency ablation. Circulation 1999; 100: $1879-1886$.

3. Haissaguerre M, Jais P, Shah DC, Garrigue S, Takahashi A, Lavergne $\mathrm{T}$, et al. Electrophysiological end point for catheter ablation of atrial fibrillation initiated from multiple pulmonary venous foci. Circulation 2000; 101: 1409-1417.

4. Yamane T, Date T, Kanzaki Y, Inada K, Matsuo S, Shibayama K, et al. Segmental pulmonary vein antrum isolation using the "large-size" lasso catheter in patients with atrial fibrillation. Circ J 2007; 71: $753-760$.

5. Jais P, Hocini M, Hsu LF, Sanders P, Scavee C, Weerasooriya R, et al. Technique and results of linear ablation at the mitral isthmus. Circulation 2004; 110: 2996-3002.

6. Hocini M, Jais P, Sanders P, Takahashi Y, Rotter M, Rostock T, et al. Techniques, evaluation, and consequences of linear block at the left atrial roof in paroxysmal atrial fibrillation: A prospective randomized study. Circulation 2005; 112: 3688-3696.

7. Miyazaki S, Kuwahara T, Takahashi A, Kobori A, Takahashi Y, Nozato T, et al. Effect of left atrial ablation on the quality of life in patients with atrial fibrillation. Circ J 2008; 72: 582-587.

8. Kumagai K, Muraoka S, Mitsutake C, Takashima H, Nakashima H. A new approach for complete isolation of the posterior left atrium including pulmonary veins for atrial fibrillation. J Cardiovasc Elec- 
trophysiol 2007; 18: 1047-1052.

9. Saksena S, Skadsberg ND, Rao HB, Filipecki A. Biatrial and threedimensional mapping of spontaneous atrial arrhythmias in patients with refractory atrial fibrillation. $J$ Cardiovasc Electrophysiol 2005; 16: $494-504$.

10. Schneider MA, Ndrepepa G, Zrenner B, Karch MR, Schreieck J, Deisenhofer I, et al. Noncontact mapping-guided catheter ablation of atrial fibrillation associated with left atrial ectopy. J Cardiovasc Electrophysiol 2000; 11: 475-479.

11. Sra J, Zaidi ST, Krum D, Georgakopoulos N, Ahmad A, Akhtar M. Correlation of spontaneous and induced premature atrial complexes initiating atrial fibrillation in humans: Electrophysiologic parameters for guiding therapy. J Cardiovasc Electrophysiol 2001; 12: 1347 1352.

12. Hindricks G, Kottkamp H. Simultaneous noncontact mapping of left atrium in patients with paroxysmal atrial fibrillation. Circulation 2001; 104: 297-303.

13. Schilling RJ, Peters NS, Davies DW. Simultaneous endocardial mapping in the human left ventricle using a noncontact catheter: Comparison of contact and reconstructed electrograms during sinus rhythm. Circulation 1998; 98: 887-898.

14. Gornick CC, Adler SW, Pederson B, Hauck J, Budd J, Schweitzer J. Validation of a new noncontact catheter system for electroanatomic mapping of left ventricular endocardium. Circulation 1999; 99: 829835 .

15. Higa S, Tai CT, Lin YJ, Liu TY, Lee PC, Huang JL, et al. Focal atrial tachycardia: New insight from noncontact mapping and catheter ablation. Circulation 2004; 109: 84-91.

16. Markides V, Schilling RJ, Ho SY, Chow AW, Davies DW, Peters NS. Characterization of left atrial activation in the intact human heart.
Circulation 2003; 107: 733-739.

17. Weber S, Ndrepepa G, Schneider M, Geissler B, Schreieck J, Karch $\mathrm{M}$, et al. Characterization of onset mechanism and waveform analysis in patients with atrial fibrillation using a high-resolution noncontact mapping system. J Cardiovasc Electrophysiol 2003; 14: 176-181.

18. Pak HN, Hwang C, Lim HE, Kim JW, Lee HS, Kim YH. Electroanatomic characteristics of atrial premature beats triggering atrial fibrillation in patients with persistent versus paroxysmal atrial fibrillation. J Cardiovasc Electrophysiol 2006; 17: 818-824.

19. Mansour M, Mandapati R, Berenfeld O, Chen J, Samie FH, Jalife J. Left-to-right gradient of atrial frequencies during acute atrial fibrillation in the isolated sheep heart. Circulation 2001; 103: 2631-2636.

20. Lazar S, Dixit S, Marchlinski FE, Callans DJ, Gerstenfeld EP. Presence of left-to-right atrial frequency gradient in paroxysmal but not persistent atrial fibrillation in humans. Circulation 2004; 110: 3181 3186.

21. Nademanee K, McKenzie J, Kosar E, Schwab M, Sunsaneewitayakul B, Vasavakul T, et al. A new approach for catheter ablation of atrial fibrillation: Mapping of the electrophysiologic substrate. J Am Coll Cardiol 2004; 43: 2044-2053.

22. Earley MJ, Abrams DJ, Sporton SC, Schilling RJ. Validation of the noncontact mapping system in the left atrium during permanent atrial fibrillation and sinus rhythm. J Am Coll Cardiol 2006; 48: 485-491.

23. Schilling RJ, Kadish AH, Peters NS, Goldberger J, Davies DW. Endocardial mapping of atrial fibrillation in the human right atrium using a non-contact catheter. Eur Heart J 2000; 21: 550-564.

24. Lin YJ, Higa S, Kao T, Tso HW, Tai CT, Chang SL, et al. Validation of the frequency spectra obtained from the noncontact unipolar electrograms during atrial fibrillation. J Cardiovasc Electrophysiol 2007; 18: $1147-1153$. 